\title{
Administration of a 20-Hydroxyeicosatetraenoic Acid Synthesis Inhibitor Improves Outcome in a Rat Model of Pediatric Traumatic Brain Injury
}

\author{
Shiyu Shu ${ }^{\mathrm{a}}$ Zhi Zhang $^{\mathrm{a}}$ Dawn Spicer $^{\mathrm{a}}$ Ewa Kulikowicz $^{\mathrm{a}} \mathrm{Ke} \mathrm{Hu}^{\mathrm{b}}$ \\ Savalan Babapoor-Farrokhran ${ }^{b}$ Sujatha Kannan ${ }^{a, c}$ Raymond C. Koehler ${ }^{a}$ \\ Courtney L. Robertson ${ }^{\mathrm{a}, \mathrm{c}}$ \\ ${ }^{a}$ Department of Anesthesiology and Critical Care Medicine, Johns Hopkins University, School of Medicine, \\ Baltimore, MD, USA; ${ }^{b}$ Department of Ophthalmology, Retina Division, Wilmer Eye Institute, Johns Hopkins \\ University, School of Medicine, Baltimore, MD, USA; ' Department of Pediatrics, Johns Hopkins University, \\ School of Medicine, Baltimore, MD, USA
}

\section{Keywords}

20-Hydroxyeicosatetraenoic acid · N-hydroxy-N-4-butyl-2methylphenylformamidine $\cdot$ Microglia $\cdot$ Neuroinflammation

\begin{abstract}
The arachidonic acid pathway metabolite 20-hydroxyeicosatetraenoic acid (20-HETE) contributes to ischemia/reperfusion brain injury. Inhibition of 20-HETE formation can protect the developing brain from global ischemia. Here, we examined whether treatment with the 20-HETE synthesis inhibitor $\mathrm{N}$-hydroxy-N-4-butyl-2-methylphenylformamidine (HET0016) can protect the immature brain from traumatic brain injury (TBI). Male rats at postnatal day 9-10 underwent controlled cortical impact followed by intraperitoneal injection with vehicle or HET0016 $(1 \mathrm{mg} / \mathrm{kg}, 5 \mathrm{~min}$ and $3 \mathrm{~h}$ postinjury). HET0016 decreased the lesion volume by over $50 \%$ at 3 days of recovery, and this effect persisted at 30 days as the brain matured. HET0016 decreased peri-lesion gene expression of proinflammatory cytokines (tumor necrosis factor-a
\end{abstract}

S.S. and C.L.R. are co-corresponding authors.

\section{KARGER}

() 2019 S. Karger AG, Basel

karger@karger.com

www.karger.com/dne
[TNF- $\alpha$ ], interleukin-1 $[$ [IL-1 $\beta]$ ) at 1 day and increased reparative cytokine (IL-4, IL-10) expression at 3 days. It also partially preserved microglial ramified processes, consistent with less activation. HET0016 decreased contralateral hindlimb foot faults and improved outcome on the novel object recognition memory task 30 days after TBI. In cultured BV2 microglia, HET0016 attenuated the lipopolysaccharide-evoked increase in release of TNF-a. Our data show that HET0016 improves acute and long-term histologic and functional outcomes, in association with an attenuated neuroinflammatory response after contusion of an immature rat brain.

○ 2019 S. Karger AG, Basel

\section{Introduction}

Worldwide, traumatic brain injury (TBI) remains the leading cause of injury-related death and disability among children [1]. Young persons who survive moderate-to-

Courtney L. Robertson, MD

Department of Anesthesiology and Critical Care Medicine

Johns Hopkins Hospital, Charlotte R. Bloomberg Children's Center, Room 6321 1800 Orleans Street, Baltimore, MD 21287 (USA)

E-Mail crober48@jhmi.edu

Shiyu Shu, MD, PhD

Department of Anesthesiology

Eye and ENT Hospital of Fudan University

No. 83, Fenyang Road, Xuhui District, Shanghai 200031 (China)

E-Mail shushiyu@hotmail.com 
severe TBI may be left with neurologic and cognitive impairment and bear the burden of these deficits lifelong [2, $3]$. Indeed, mortality and morbidity are worse in infants and toddlers than in older children [1]. Given that the human brain continues to mature into the early third decade, early childhood TBI can influence later brain development [4]. Thus, it is essential to explore the basic mechanisms and potential treatments for TBI in the immature brain.

The initial direct mechanical injury is considerably worsened by secondary cascades that activate many different signaling pathways, such as an enhanced inflammatory response, mitochondrial dysfunction, bloodbrain barrier dysfunction, edema formation, delayed cell death, gliosis, and cerebral cavity formation [5-7]. Treatments to combat the harmful secondary cascades would be advantageous for the recovery of the immature brain, whose mechanisms and responses to TBI can differ from those in the adult brain $[6,8-10]$. Only a modest number of pediatric clinical trials for TBI have been conducted, and thus far, nearly all have failed to show a significant benefit of any specific therapy [11].

20-Hydroxyeicosatetraenoic acid (20-HETE) has been shown to contribute to ischemia/reperfusion injury in brain [12], heart [13], and kidney [14]. It is synthesized by members of the cytochrome P450 (CYP) 4A family and is the $\omega$-hydroxylation product of arachidonic acid, which can be mobilized from the phospholipid pool after both ischemia and TBI [15]. CYP4A has been detected in cerebral vascular smooth muscle $[16,17]$, neurons [18, 19], cultured astrocytes [20], and postischemic microglia [21]. Moreover, 20-HETE can exert direct effects on key neuronal proteins involved in excitotoxicity in vivo [22] and contribute to neurodegeneration after oxygen-glucose deprivation in hippocampal slices and cultured neurons [23]. This evidence suggests that some of its action is nonvascular. Inhibition of 20 HETE has been shown to reduce infarct volume and provides neuroprotection in models of ischemic and hemorrhagic stroke in rats and nonhuman primates [12, 24-27]. Importantly, in the developing brain, 20-HETE inhibition reduced markers of oxidative stress and improved neuronal survival and neurologic outcome in a piglet cardiac arrest model [22]. The secondary injury mediators and time course of injury can differ between ischemic brain injury and TBI. However, there are several potential overlaps, such as blood brain barrier breakdown, robust neuroinflammation, and oxidative stress. It is known that TBI induces arachidonic acid release from cell membranes, with both early elevations and sustained levels seen in animal and human brain after TBI $[28,29]$. Additionally, CYP4A and 4F gene expression increases in hippocampus and parietal cortex from $6 \mathrm{~h}$ to 7 day after controlled cortical impact (CCI) in adult rats [30]. Very recently, significant elevations in 20-HETE were demonstrated in brain following CCI in adult rats [31], and inhibition of 20-HETE improved blood brain barrier integrity and reduced brain edema [32].

In addition to effects on neuronal cell death and neurodegeneration, 20-HETE may play an important role in other cell types. CYP4A has been detected in cultured astrocytes [20] and post-ischemic microglia [21]. Given the robust role for neuroinflammation in the pathobiology of TBI, especially in the developing brain, we chose to specifically investigate the relationship between 20HETE inhibition and microglial activation in this study. Because TBI can share common mechanisms with ischemic brain injury, and with emerging studies suggesting a protective role for 20-HETE inhibition after TBI, we designed this study to examine the effect 20-HETE inhibition in a pediatric rat CCI model. We chose to use Nhydroxy-N-4-butyl-2-methylphenylformamidine (HET0016), a specific inhibitor of 20-HETE synthesis [33], that protects the immature [22] and mature [12] brain from ischemic/reperfusion injury, to determine whether it protects against neuroinflammation caused by TBI. We used rats at postnatal day (PND) 9-10 to model infant/toddler TBI.

\section{Methods and Materials}

\section{Model}

All experimental protocols were approved by the Animal Care and Use Committee of the Johns Hopkins University and were performed in accordance with National Institutes of Health guidelines. Male Sprague-Dawley rats (Charles River; PND 9-10, 18$22 \mathrm{~g}$ ) were used in the study and housed with littermates both before surgery and after recovery from anesthesia.

Surgical anesthesia was induced with $4 \%$ isoflurane inhalation. Once anesthetized, animals were secured in a stereotaxic device and administered maintenance anesthesia (2\% isoflurane) with $30 \%$ oxygen via nose cone. A rectal probe and heating blanket were used to maintain a rectal temperature of $37.0 \pm 0.5^{\circ} \mathrm{C}$. A midline incision was made over the skull, and a high-speed dental drill was used to create a left parietal craniotomy. CCI injury was generated with a $3-\mathrm{mm}$ flat-tipped impactor at $5.5 \mathrm{~m} / \mathrm{s}$ velocity, $50 \mathrm{~ms}$ duration, and a dural displacement of $1.5 \mathrm{~mm}[34,35]$. After the impact, the craniotomy was resealed with an acrylic mixture, and the surgical site was closed with interrupted sutures. After surgery was complete, anesthesia was discontinued, and the rat was awakened and returned to the dam and littermates. 


\section{Experimental Groups}

Within each litter, 2-4 rats were randomly assigned to 1 of 3 groups in a balanced fashion as follows: age-matched, naïve littermate group without anesthesia or surgery, vehicle-treated CCI group, and HET0016-treated CCI group. Within each litter, surgery was done over 2 consecutive days (PND 9 and 10). The precise order of treatment was not randomized, but the order of treatment varied among litters. This was done to minimize the effect on outcome of the between-litter variance. Because the primary hypothesis to be tested here was whether HET0016 reduced injury from CCI, we used naïve littermates as the uninjured control group. This is especially important for functional outcome testing, as we have recently noted in other cohorts that the craniotomy at this very young age produced some loss of underlying cortex and mild functional deficits, when the rats were survived for 4 months (unpublished).

HET0016 (Cayman Chemical Company, Ann Arbor, MI, USA) was administered at a dose of $1 \mathrm{mg} / \mathrm{kg}$ based on previous work in immature animal cardiac arrest studies [22] and in adult focal ischemia [12]. This dose inhibited production of 20-HETE in rat cortex without inhibiting production of epoxyeicosatrienoic acids or their dihydroxyeicosatetraenoic acid metabolites [36]. Drug or vehicle was injected intraperitoneally at $5 \mathrm{~min}$ and again at $3 \mathrm{~h}$ after CCI. The timing of this dosing was based on previous work showing neuroprotection with early dose initiation after focal and global ischemia $[12,37,38]$. The vehicle consisted of $45 \%$ (2-hydroxypropyl)- $\beta$-cyclodextrin in saline.

\section{Tissue Processing}

Rats were anesthetized with an overdose of pentobarbital sodium $(120 \mathrm{mg} / \mathrm{kg})$. For histology, immunohistochemistry, and immunofluorescence analysis, rats were perfused transcardially with ice-cold phosphate-buffered saline and $4 \%$ paraformaldehyde before their brains were removed and dehydrated in a 10-20-30\%-gradient sucrose solution and frozen for cryostat sectioning in optimal cutting temperature embedding medium. For gene expression measurements, rats were killed by decapitation, and brain samples were harvested and kept in RNAlater (Thermo Scientific, Waltham, MA, USA) at $-20{ }^{\circ} \mathrm{C}$. For protein expression measurement, the brain was removed after the transcardial perfusion with cold phosphate-buffered saline and then stored at $-80^{\circ} \mathrm{C}$.

To assess lesion volume, we stained $40-\mu \mathrm{m}$ coronal sections of brain with Cresyl violet. We determined the area of intact tissue in the ipsilateral and contralateral hemisphere of every 24 th section with ImageJ software. The intact hemisphere volume was obtained by multiplying the sum of the intact hemisphere areas by the distance between sections. The lesion volume was calculated by taking the difference in the volumes between the intact contralateral and ipsilateral hemispheres. The lesion volume was expressed as a percent of the contralateral hemisphere volume.

\section{Foot Fault Test}

Investigators blinded to the experimental groups tested rats at 28-30 days after CCI; age-matched naïve littermates were also tested. The rats were placed on an elevated horizontal metal grid with a mirror underneath for video recording of their paws, each of which was painted with a different color for ease of identification. The number of foot slips for each limb over $10 \mathrm{~min}$ was analyzed during the video playback.

\section{Novel Object Recognition}

At approximately 25 days after CCI, an investigator blinded to the experimental groups tested CCI rats and age-matched littermates in the novel object recognition test. Rats were placed individually in an empty plastic box $(0.4 \times 0.4 \mathrm{~m})$ for $5 \mathrm{~min}$ on 3 consecutive days to acclimate them to the chamber. On the fourth day, rats were placed in the same box with 2 objects of identical shape and color for $5 \mathrm{~min}$ for familiarization and memory encoding. Twenty-four hours later, the rats were again placed in the box, but one of the familiar objects was replaced with a novel object of different color and shape. The amount of time each rat interacted with the novel object and the familiar object was analyzed on video playback. Near direct contact with nose or whiskers was considered an interaction. A discrimination index was calculated by subtracting the time spent interacting with the familiar object from the time spent interacting with the novel object; the difference was divided by the total interaction time over the first $30 \mathrm{~s}$ after being placed in the chamber.

\section{Microglial Staining}

To study microglial activation, we used monoclonal anti-ionized calcium-binding adapter molecule-1 (Iba-1) rabbit antibody (\#019-19741, Wako Pure Chemical Industries, Ltd., Japan). Tissue sections were incubated with the primary antibody overnight at $4^{\circ} \mathrm{C}$. A biotinylated universal secondary antibody was then applied for $1 \mathrm{~h}$ (anti-rabbit IgG, Vector Labs, Inc., Burlingame, CA, USA), followed by an avidin biotin complex according to the manufacturer's instructions (Vectastain Universal Elite Kit, Vector Labs). We visualized the antibody binding using the 3,3'-diaminobenzidine peroxidase substrate kit (Vector Labs). We counterstained with hematoxylin and examined the sections using light microscopy on a Leica DMRB microscope (Leica Microsystems, Bannockburn, IL, USA).

\section{Microglial Morphological Analysis}

All slides and images were coded, and the analysis was performed with the personnel blinded to experiments. The microscopic fields (4-5/animal) to be analyzed for microglia morphology were chosen in an unbiased fashion from the peri-lesion area using Nikon Eclipse 90i and Stereo Investigator software (MBF Bioscience, Williston, VT, USA). The peri-lesion area was defined as an area of cortex adjacent to the edges of the lesion, in the central section of the traumatic injury. The peri-lesion area was identified at low power $(10 \times)$, selecting a consistent area between rats. The microscope was then moved to high power $(40 \times)$ and microglia within that high-powered field that met criteria were traced. The microglia that met the following criteria were traced: (1) cell body and processes completely contained within the slice; (2) cells sufficiently stained to allow for tracing processes. Using these criteria, there were generally 2 microglia/field that were traced. The soma and processes morphology of the microglia were analyzed using the Neurolucida and Neurolucida Explorer software package (MBF Bioscience, Williston, VT, USA). There were 4-5 fields/animal, with $\sim 2$ microglia/field, for a total of 8-10 microglia/animal being traced.

RNA Isolation and Quantitative Real-Time Polymerase Chain Reaction

Quantitative real-time polymerase chain reaction (PCR) was used to analyze mRNA expression of the following inflammatory 


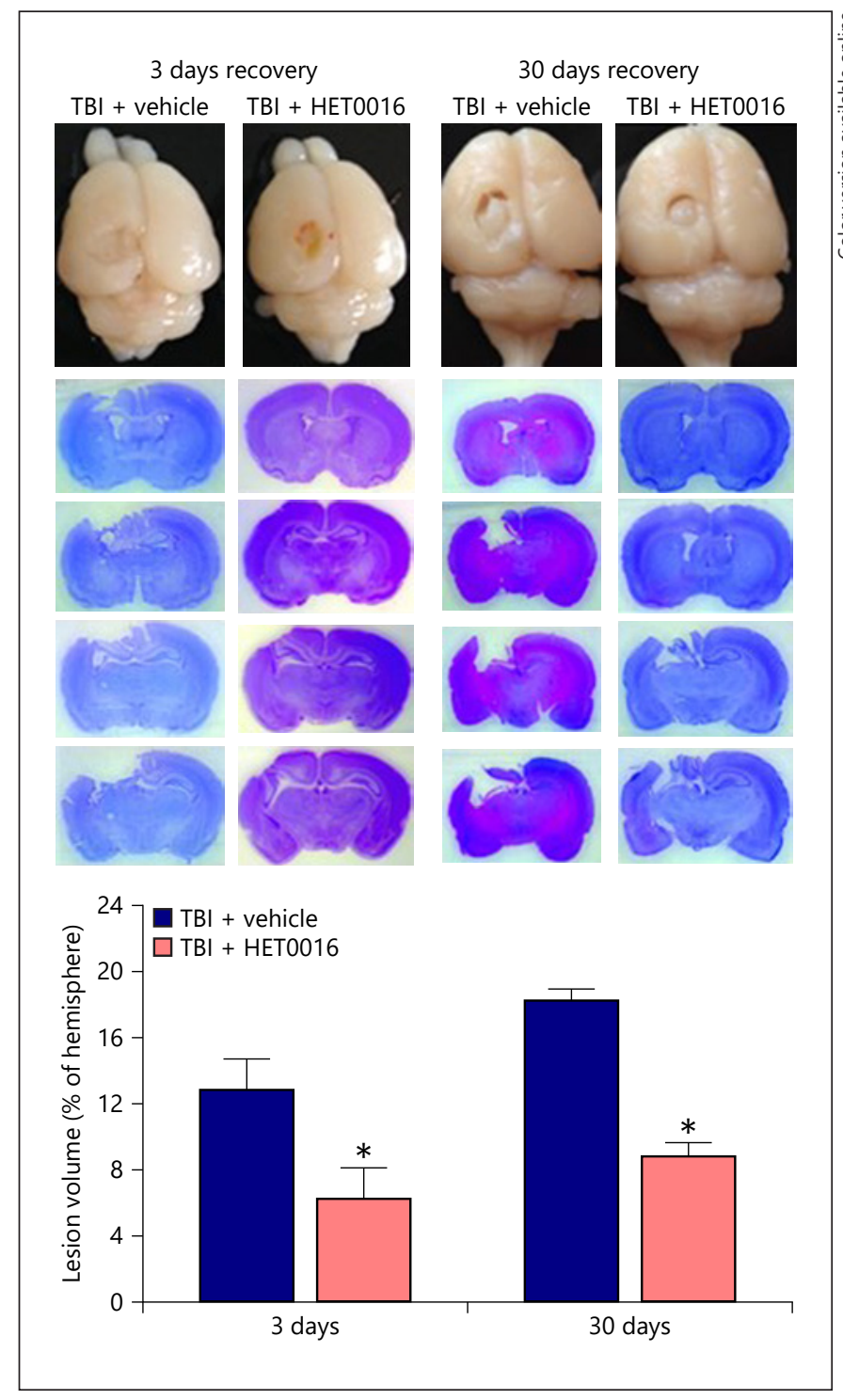

Fig. 1. Representative images of brain lesions from the vehicletreated and HET0016-treated rats on days 3 and 30 after CCI. Serial coronal sections of one representative animal/group are displayed. Bar graph shows the mean \pm SD of the lesion volume as a percentage of the corresponding contralateral hemisphere volume on day 3 (vehicle, $n=10$; HET0016, $n=10$ ) and day 30 (vehicle, $n=10$; HET0016, $n=7) .{ }^{*} p<0.05$ versus the corresponding vehicle group ( $t$ test). TBI, traumatic brain injury; HET0016, N-hydroxy-N-4-butyl-2-methylphenylformamidine.

markers in rat brains from each experimental group: tumor necrosis factor- $\alpha$ (TNF- $\alpha$ ), interleukin-1 $\beta$ (IL-1 $\beta$ ), IL-4, and IL-10. Total RNA was isolated from brain tissue using TRIzol reagent (Invitrogen, Grand Island, NY, USA). cDNA was synthesized from $1 \mu \mathrm{g}$ total RNA with the Sensiscript Reverse Transcriptase Kit (Qiagen, Valencia, CA, USA). Quantitative real-time PCR was performed on an ABI 7500 system using Power SYBR green (Applied Biosys-
Table 1. Primer sequences used in real-time PCR

\begin{tabular}{|c|c|c|}
\hline Gene & Primer sequences & $\begin{array}{l}\text { Product } \\
\text { size }\end{array}$ \\
\hline GADPH & $\begin{array}{l}\text { 5'-GCAAGAGAGAGGCCCTCAG-3' } \\
\text { 5'-TGTGAGGGAGATGCTCAGTG-3' }^{\prime}\end{array}$ & 74 \\
\hline IL- $1 \beta$ & $\begin{array}{l}\text { 5'-CACCTCTCAAGCAGAGCACAG-3' } \\
5^{\prime} \text {-GGGTTCCATGGTGAAGTCAAC-3' }\end{array}$ & 79 \\
\hline TNF- $\alpha$ & $\begin{array}{l}\text { 5'-CCAGGAGAAAGTCAGCCTCCT-3' } \\
5^{\prime} \text {-TCATACCAGGGCTTGAGCTCA-3' }\end{array}$ & 87 \\
\hline IL-4 & $\begin{array}{l}5^{\prime} \text {-TCCTTACGGCAACAAGGAAC-3' } \\
5^{\prime} \text {-GTGAGTTCAGACCGCTGACA-3' }\end{array}$ & 181 \\
\hline IL-10 & $\begin{array}{l}\text { 5'-CACTGCTATGTTGCCTGCTC-3' } \\
5^{\prime} \text {-TTCATGGCCTTGTAGACACC-3' }\end{array}$ & 463 \\
\hline
\end{tabular}

GADPH, glyceraldehyde 3-phosphate dehydrogenase; IL-1 $\beta$, interleukin-1 $\beta$; TNF- $\alpha$; tumor necrosis factor- $\alpha$; PCR, polymerase chain reaction.

Table 2. Group sample sizes in outcomes studied

\begin{tabular}{lccc}
\hline \multirow{2}{*}{ Outcome } & \multicolumn{3}{l}{ Group sample size } \\
\cline { 2 - 4 } & naïve & TBI + & TBI + \\
& & Vehicle & HET0016 \\
\hline Lesion volume 3 days & 5 & 10 & 10 \\
Lesion volume 30 days & 5 & 10 & 7 \\
Foot fault & 11 & 15 & 23 \\
Novel object & 11 & 14 & 23 \\
Microglia 3 days & 8 & 8 & 8 \\
Microglia 30 days & 4 & 17 & 10 \\
Cytokine 1 day & $\mathrm{n} / \mathrm{a}$ & 4 & 4 \\
Cytokine 10 days & $\mathrm{n} / \mathrm{a}$ & 10 & 10
\end{tabular}

TBI, traumatic brain injury; HET0016, N-hydroxy-N-4-butyl2-methylphenylformamidine.

tems, Carlsbad, CA, USA). The primer sequences are shown in Table 1. Ct values from triplicate measurements of each sample were averaged, and relative expression levels were determined by the $\Delta \Delta \mathrm{Ct}$ method, with glyceraldehyde 3-phosphate dehydrogenase serving as the reference gene. Values were normalized by concurrently run mRNA values of naïve littermates that did not undergo surgery but which had their brains harvested on the same PND.

\section{Cell Culture}

The immortalized BV2 murine microglia cell line was maintained in Dulbecco's modified Eagle's medium supplemented with $10 \%$ fetal bovine serum, $100 \mathrm{U} / \mathrm{mL}$ penicillin, and $100 \mu \mathrm{g} / \mathrm{mL}$ streptomycin at $37^{\circ} \mathrm{C}$ in an atmosphere of $5 \% \mathrm{CO}_{2}$ in air. BV2 microglia were divided into the following treatment groups: control, HET0016 (0.1 $\mu \mathrm{M})$, HET0016 (1 $\mu \mathrm{M})$, lipopolysaccharide (LPS; 50 
Fig. 2. HET0016 treatment improves sensorimotor and cognitive outcome in rats at 1 month after CCI. a Number of contralateral hindlimb foot faults on a horizontal grid in rats treated with vehicle $(n=15)$ or HET0016 $(n=23)$ after CCI and in naïve age-matched littermates $(n=11)$. b Discrimination index in novel object recognition test of CCI rats treated with vehicle $(n=14)$ or HET0016 $(n=23)$ and of naïve age-matched littermates $(n=11)$. Zero represents equal time interacting with the novel and familiar object. Data are shown as box plots of 25,50 , and 75 percentiles and whiskers show the 5 and 95 percentiles; $* p<0.05$ versus naïve group; ${ }^{\dagger} p<0.05$ versus vehicle group (Kruskal-Wallis analysis of ranks followed by pairwise comparisons with the Mann-Whitney test). CCI, controlled cortical impact; HET0016, N-hydroxy-N-4-butyl-2-methylphenylformamidine.

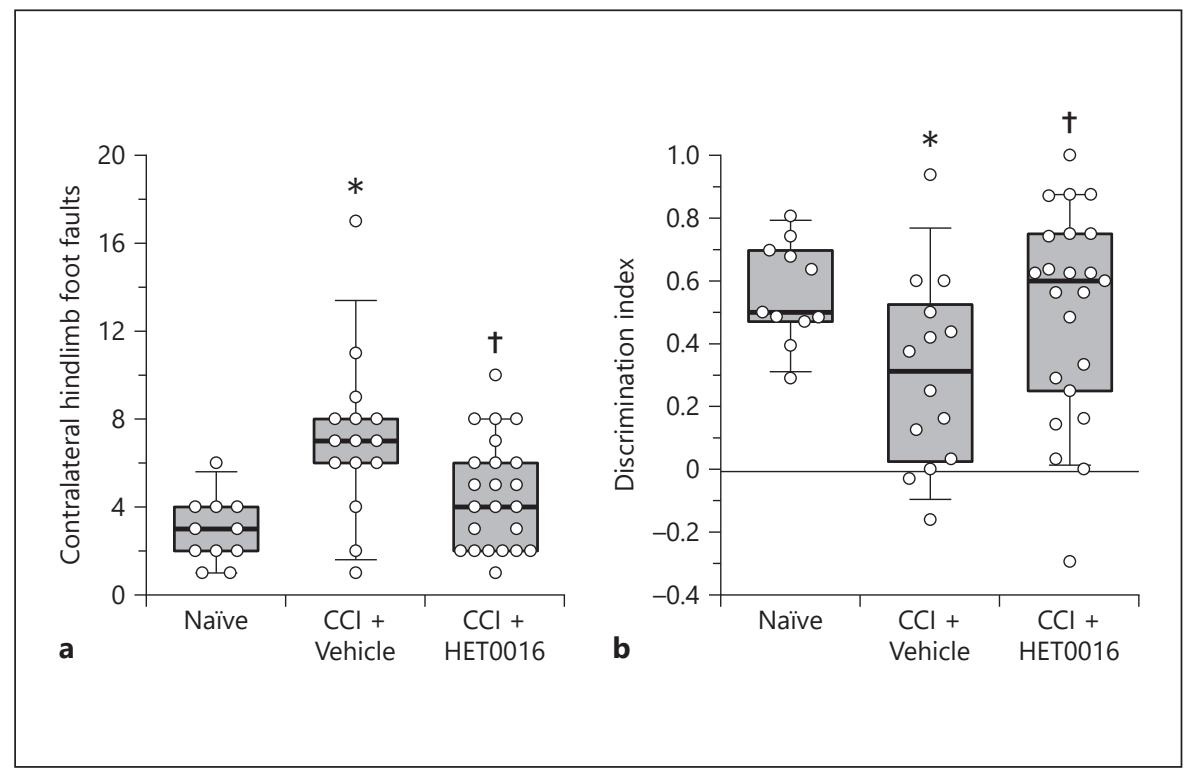

$\mathrm{ng} / \mathrm{mL})$, LPS + HET0016 $(0.1 \mu \mathrm{M})$, and LPS + HET0016 (1 $\mu \mathrm{M})$. Mouse TNF- $\alpha$ ELISA kit (Cat. No. 430907, BioLegend, San Diego, CA, USA) was used to measure the TNF- $\alpha$ expression in the cell culture medium.

\section{Statistical Analysis}

The results are expressed as mean $\pm \mathrm{SD}$. Based on previous work, we expected a sample size of 10 to provide $80 \%$ power for detecting a $25 \%$ change in lesion volume relative to vehicle values, with naïve (uninjured) controls only requiring a sample size of 5 [35]. Total animal numbers in each group studied are found in Table 2. Statistical analysis was performed with GraphPad Prism software (GraphPad Software, Inc., La Jolla, CA, USA, version 5.0). Comparisons of multiple groups were performed by one-way analysis of variance followed by Bonferroni's correction for multiple comparisons. When normality or equal variance tests failed, the Kruskal-Wallis analysis of ranks test was used, and individual group comparisons were made with the Mann-Whitney test. A $p$ value of $<0.05$ was considered statistically significant in all tests.

\section{Results}

\section{HET0016 Reduced Brain Lesion Volume}

We evaluated lesion volume on days 3 and 30 after CCI. Examples of Cresyl violet-stained sections are shown in Figure 1, with serial sections from one representative animal from each group displayed. Lesion volume at 3 days of recovery was significantly smaller after HET0016 treatment $(6.2 \pm 1.9 \%$ of contralateral volume; $n=10)$ than after vehicle treatment $(12.9 \pm 1.9 \% ; n=10, p<0.05)$. After 30 days of recovery (PND 39), lesion volume enlarged to $18.2 \pm 0.8 \%$ of the contralateral hemisphere vol- ume in the vehicle-treated group $(n=10)$. Over this 30 day time span, hemisphere volume in naïve age-matched controls was found to increase by $63 \%$. Thus, despite the large brain growth that occurred in the rats between PNDs 12 and 39, the lesion volume grew disproportionately more than the rest of the brain in the vehicle-treated group $(12.9 \pm 1.9 \%$ to $18.2 \pm 0.8 \%$ of the contralateral hemisphere volume). With HET0016 treatment, lesion volume enlarged to only $8.8 \pm 0.9 \%(n=7)$ of the contralateral hemisphere volume at 30 days of recovery, and this value remained significantly less than that in the vehicletreated group $(p<0.05)$.

\section{HET0016 Led to Improved Sensorimotor and Cognitive Function Later in Development}

At 1 month after CCI, we examined whether neuroprotection led to improved sensorimotor and cognitive function later in development. When rats were placed on a horizontal grid for $10 \mathrm{~min}$, the number of foot faults of the hindlimb contralateral to the injury was greater in the CCI rats than in the age-matched littermates (Fig. 2a). In contrast, HET0016 treatment significantly reduced foot faults in CCI rats to a number that was comparable to that in the naïve group. With the novel object recognition test, the naïve rats spent more time interacting with the novel object, as indicated by a positive discrimination index (Fig. 2b). In the vehicle-treated CCI group, this discrimination index was significantly decreased. Treatment with HET0016 after CCI significantly increased the index compared to that in the vehicle-treated group. Thus, ear- 


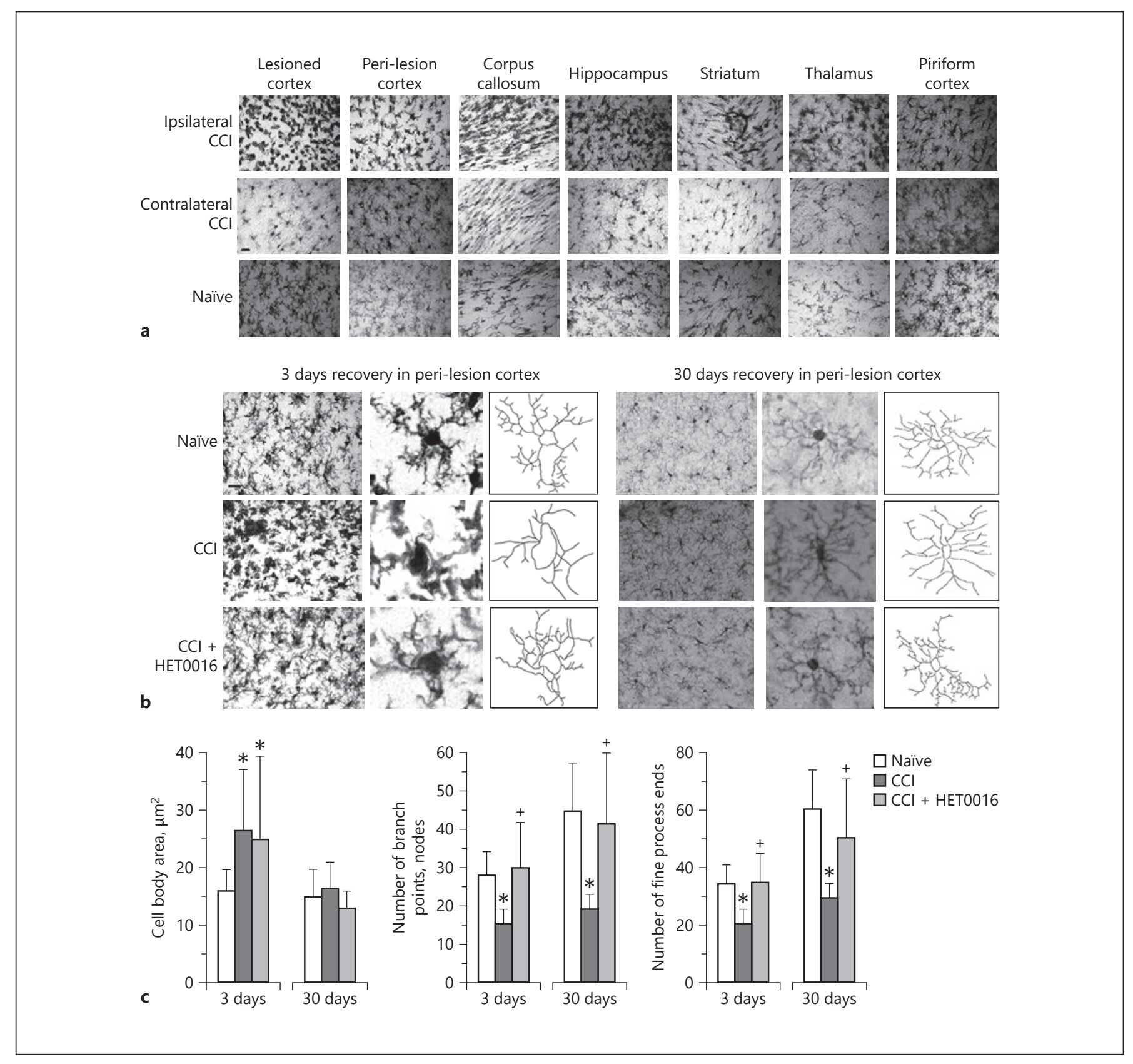

Fig. 3. Morphologic changes of microglia after CCI. a At 3 days after CCI in rats, immunohistochemical staining for Iba- 1 revealed a change in microglia morphology consisting of increased cell body size and less ramification of processes throughout the hemisphere ipsilateral to the injury relative to that in the corresponding contralateral regions and age-matched naïve brain. Scale bar $=20$ $\mu \mathrm{m}$. b Images of Iba-1-stained microglia in peri-lesion cortex at 3 and 30 days after CCI and in naïve rat cortex. Scale bar $=20 \mu \mathrm{m}$. Morphometric analysis of high-power images of microglial cell bodies and processes with Neurolucida software indicated that loss of process complexity persisted in vehicle-treated rats 30 days after
CCI, whereas process complexity was better retained at 3 and 30 days in HET0016-treated rats. c Calculations of microglial cell body area, number of cell process branch points, and number of fine end processes (mean \pm SD) in rat peri-lesion cortex at 3 and 30 days in naïve ( 3 days, $n=8 ; 30$ days, $n=3)$, CCI + vehicle $(3$ days, $n=8$; 30 days, $n=17$ ), and CCI + HET0016 ( 3 days, $n=8$; 30 days, $n=10)$ groups. ${ }^{*} p<0.05$ versus naïve group; ${ }^{+} p<0.05$ versus CCI + vehicle group (ANOVA and Bonferroni correction for multiple comparisons). CCI, controlled cortical impact; HET0016, N-hydroxy-N-4-butyl-2-methylphenylformamidine. 


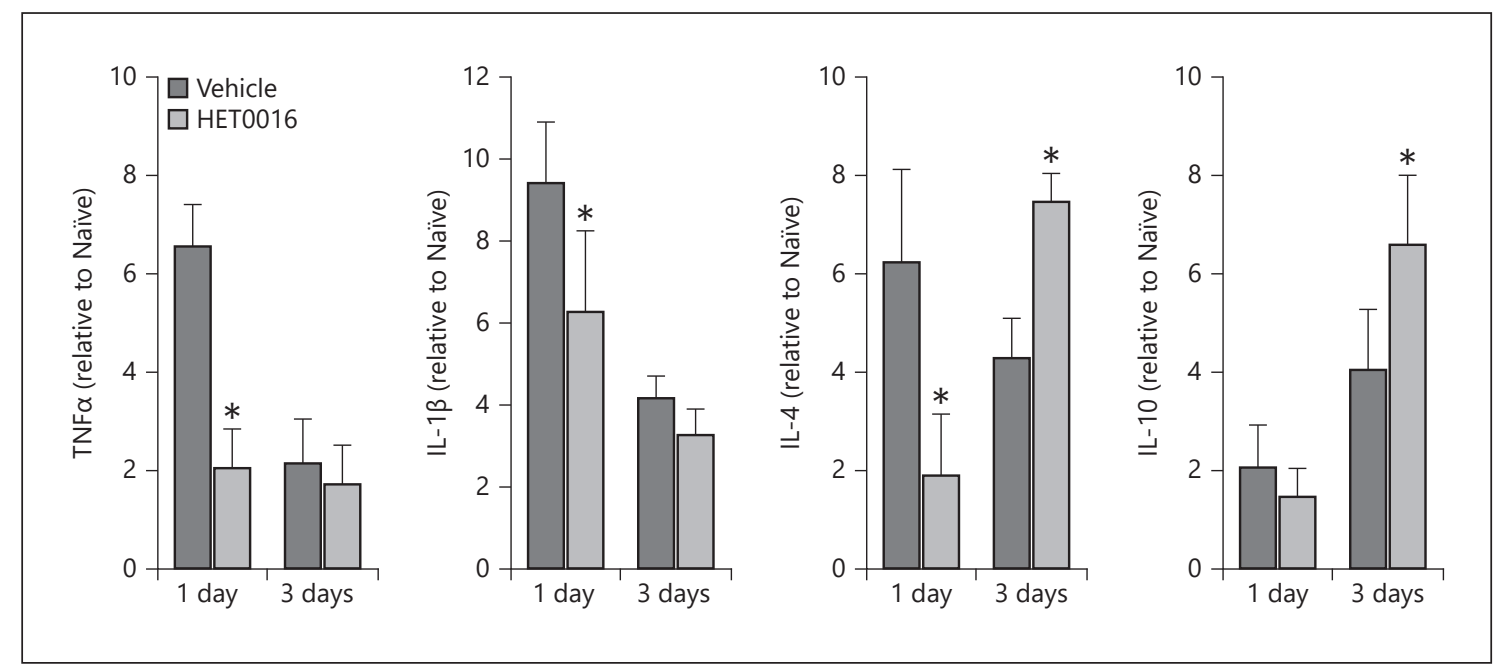

Fig. 4. TNF- $\alpha$, IL-1 $\beta$, IL-4, and IL-10 mRNA levels measured by quantitative real-time PCR in ipsilateral hemisphere 1 day after CCI + vehicle $(n=4)$, or CCI + HET0016 $(n=4)$ and 3 days after CCI + vehicle $(n=10)$, or CCI + HET0016 $(n=10)$. Values are expressed as fold changes from age-matched naïve littermates $(\operatorname{mean} \pm \mathrm{SD})$. $* p<0.05$ versus CCI + vehicle at the same time point $(t$ test). TNF- $\alpha$, tumor necrosis factor- $\alpha$; HET0016, Nhydroxy-N-4-butyl-2-methylphenylformamidine; IL-1 $\beta$, interleukin-1 $\beta$.

ly administration of HET0016 after CCI offered longterm protection of sensorimotor and cognitive function.

\section{HET0016 Reduced Microglial Activation after TBI}

When they are activated, microglia are well known to take on an amoeboid morphology, with enlargement of the cell body and retraction of processes. The morphology of Iba-1-stained microglia indicated widespread activation in cortex, corpus callosum, hippocampus, striatum, thalamus, and piriform cortex 3 days after CCI (Fig. 3a). Thus, microglial activation was not limited to the peri-lesion region. To quantify the effects of HET0016 treatment, we focused on peri-lesion cortex, where changes in microglial morphology were profound. We observed enlargement of the microglial cell body 3 days after injury that was unaffected by HET0016 treatment (Fig. 3b). In contrast, the number of nodes, or branch points, and the number of ends of the fine processes were decreased in rats that underwent CCI with vehicle treatment; however, this decrease was significantly attenuated in HET0016-treated rats. By 30 days, microglial cell body area recovered to levels seen in naïve age-matched littermates (Fig. 3c). However, the number of branch points and ends of fine processes remained less in vehicle-treated group than in the naïve group. Again, HET0016 treatment maintained the branching and number of end processes at levels seen in the naïve group. Thus, 20-HETE appears to contribute to the loss of ramified processes in microglia after CCI.

\section{HET0016 Attenuated Gene Expression of}

Proinflammatory Cytokines at 1 Day and Augmented Expression of Anti-Inflammatory Cytokines at 3 Days

We also examined the neuroinflammatory response by measuring gene expression of various cytokines in the cortex after CCI (Fig. 4). At 1 day after TBI, we observed a 7- to 9-fold increase in gene expression of TNF- $\alpha$ and IL- $1 \beta$ relative to levels in the sham group. The increase in these proinflammatory cytokines was significantly decreased by HET0016. By 3 days of recovery, the increase in TNF- $\alpha$ and IL- $1 \beta$ mRNA subsided in the vehicle group, and the increased expression was no longer different from those in the HET0016-treated group. Interestingly, gene expression of the anti-inflammatory IL- 4 was attenuated at 1 day but was augmented by 3 days of recovery. In contrast, expression of the anti-inflammatory IL-10 cytokine was unaffected at 1 day and augmented at 3 days of recovery by the earlier treatment with HET0016. Thus, it appears that HET0016 generally attenuated the proinflammatory response at 1 day and augmented the antiinflammatory response at 3 days after CCI.

\section{HET0016 Blunted the LPS-Induced Expression of TNF- $\alpha$ in BV2 Microglia}

To investigate whether microglia can be directly influenced by HET0016, we treated a BV2 microglial cell line with $0,0.1,1$, or $10 \mu \mathrm{M}$ HET0016 with or without LPS for $24 \mathrm{~h}$. Previous in vivo studies have shown that systemic 


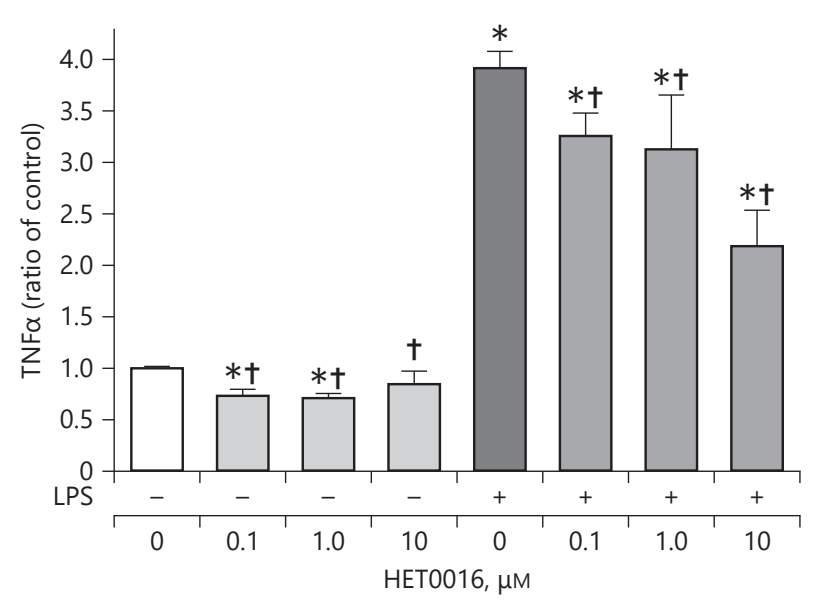

Fig. 5. LPS activation of microglial cells in vitro. Increased expression of TNF- $\alpha$ evoked by $50 \mathrm{ng} / \mathrm{mL}$ LPS in a BV2 microglial cell line was attenuated by treatment with $0.1,1$, and $10 \mu \mathrm{M}$ HET0016 $(n=4$ per group, mean $\pm \mathrm{SD}) .^{*} p<0.05$ versus control; ${ }^{\dagger} p<0.05$ versus LPS alone (ANOVA and Bonferroni correction for multiple comparisons). LPS, lipopolysaccharide; TNF- $\alpha$, tumor necrosis factor- $\alpha$; HET0016, N-hydroxy-N-4-butyl-2-methylphenylformamidine.

(IV/IP) treatment with $10 \mathrm{mg} / \mathrm{kg}$ HET0016 produces brain levels of $0.2-7.2 \mu \mathrm{M}[39,40]$. So $1 \mathrm{mg} / \mathrm{kg}$ systemic treatment should be expected to produce brain levels of $\sim 0.02-0.7 \mu \mathrm{M}$, which is relatively comparable to the 1 st 2 doses studied here $(0.1$ and $1 \mu \mathrm{M})$. We found that the increase in TNF- $\alpha$ evoked by LPS was attenuated by $0.1,1$, and $10 \mu \mathrm{M}$ HET0016 (Fig. 5). These data suggest that HET0016 might act directly on microglia in vivo to reduce the inflammatory response.

\section{Discussion}

The results of this study show that administration of the 20-HETE synthesis inhibitor HET0016 after CCI in PND 9-10 rats exerts a marked protective effect on the early development of the lesion and the expansion of the lesion over a 30-day recovery period. A smaller lesion was associated with significantly improved sensorimotor and cognitive function. Moreover, treatment with HET0016 attenuated early and late morphologic changes in perilesion microglia and early induction of proinflammatory cytokines. It also augmented delayed induction of antiinflammatory cytokines. Although the reduced inflammatory response may be secondary to reduced neuronal injury, we found that HET0016 treatment was capable of directly suppressing the TNF- $\alpha$ response of a microglia cell line to LPS. Thus, it is also possible that the suppression of the inflammatory response and growth of the lesion observed in vivo after CCI might be attributed to direct effects of HET0016 on microglia.

Lesion volume is a common outcome measure in the CCI model. However, when assessing the lesion volume in immature brain, one needs to consider the influence of natural brain growth during development. The brain in naïve rats at PNDs 12 and 39 showed the expected developmental increase in volume. However, the cavitation of the developing brain also grows in size over time after CCI. Interestingly, when lesion volume was plotted as a percent of hemisphere volume, the percentage of lesion volume increased between 3 and 30 days of recovery. Similar results have been reported between 2 weeks and 3 months of recovery from CCI in P21 mice [41]. This lesion growth suggests that neuroinflammation secondary to the initial injury may increase the lesion disproportionately more than the natural growth of the rest of the brain. With HET0016 treatment, the lesion also expanded at 30 days of recovery, but the lesion volume as a percent of the more mature and larger brain remained substantially smaller than that in the vehicle group. Functionally, this protection was reflected in statistically significant improvement in performance at 1 month of recovery on the foot-fault test and novel object recognition test. However, it should be noted that the variance remained greater than in the naïve group, suggesting that some animals still experienced a neurodevelopmental delay in these sensorimotor and cognitive tasks.

To further investigate the underlying mechanisms by which HET0016 offers protection against TBI, we investigated the relationship between HET0016 and microglial activation because microglial-mediated neuroinflammation is considered to be an important secondary injury mechanism after TBI $[42,43]$. Upon activation, microglia undergo marked changes in cell morphology; that is, they contract their processes and transform from a resting state with a ramified cellular morphology to an activated state with an amoeboid-like morphology. Activated microglia can secrete many factors, including proinflammatory and anti-inflammatory cytokines, chemokines, and neurotrophic factors that play an important role in determining the molecular phenotype and functional response of microglia after brain injury. Moreover, microglia may contribute to long-term loss of cognitive function [44], possibly mediated by microglial interactions with axons, dendrites, and synaptic organization [43]. 
We found that microglia were markedly activated on the third day after injury. Furthermore, they were activated not only in the peri-lesion cortex and nearby corpus callosum and hippocampus but also in more distant regions such as striatum, thalamus, and piriform cortex. Sublethal structural damage in distant regions could lead to diffuse microglia activation. We did not investigate the presence of a contrecoup focal lesion in piriform cortex, which could also account for distant microglia activation. Remote microglial activation in connected regions away from the site of injury has been described in patients with TBI that may persist for a prolonged period of time after the injury [45].

Using Neurolucida software for analysis, we found differences in microglial morphology in the naive cortex between PND 12 and PND 39. Whereas the cell body size was similar, the number of branch points and number of fine process ends increased, indicating a developmental increase in microglial ramification of processes. After CCI, microglial cell bodies enlarged and processes retracted, consistent with activation. Interestingly, HET0016 treatment soon after CCI altered microglial appearance. Although HET0016 did not affect the increase in cell body size seen at 3 days after injury, it did attenuate the loss of branch points and number of fine processes. Thus, HET0016 altered the microglial phenotype progression and appeared to limit activation. The cytokine response was consistent with this interpretation in that HET0016 blunted the induction of proinflammatory TNF- $\alpha$ and IL- $1 \beta$ seen at 1 day of recovery in the vehicle group. However, it also attenuated the increase in IL-4, which normally promotes the repair state of macrophages. This attenuation might reflect a diminished activation of the synchronized cytokine response secondary to diminished cell death. However, by 3 days HET0016treated rats exhibited increased induction of IL- 4 and IL10 , which is also associated with repair and phagocytosis. In general, HET0016 treatment soon after the insult ameliorated the proinflammatory response and augmented the induction of the repair process.

By 30 days post-injury, the size of microglial cell bodies had recovered in both groups, but the number of branch points and fine process ends in the vehicle group remained much less than that in the naïve group. In contrast, the number of branch points and fine process ends was fully restored in the HET0016 group. Therefore, treatment with HET0016 on the day of injury has a longlasting effect in normalizing the microglial response.

To determine whether HET0016 can inhibit microglial activation directly, we activated cultured BV2 cells with LPS. LPS produced the expected release of TNF- $\alpha$ into the media and this increase was moderately attenuated by $0.1 \mu \mathrm{M}$ HET0016. This concentration is known to inhibit 20-HETE synthesis in other cell systems. Whereas increasing the concentration to $1 \mu \mathrm{M}$ produced no additional inhibition of TNF- $\alpha$ release, the $10 \mu \mathrm{M}$ concentration did. We cannot exclude off-target effects on enzymes other than $\omega$-hydroxylase enzymes at the highest concentration [33]. Nevertheless, these data suggest that HET0016 could act directly on microglia to blunt the release of TNF- $\alpha$ and possibly other proinflammatory cytokines.

There are several potential limitations of this study. First, the primary control group was naïve littermates, and we did not include sham-operated pups. Recent work by our group has shown greater than expected tissue loss in sham (craniotomy only) pups and mild functional deficits, when survived for up to 4 months (unpublished data). Given that the primary hypothesis to be tested here was the protective effects of HET0016, we focused comparisons on vehicle versus HET0016 treated, injured rats, and used naïve littermates as the healthy, uninjured control group. With this approach, we may not fully appreciate the small component of injury attributable to the sham craniotomy or brief anesthesia exposure in these young rat pups. Another potential limitation is that control animals did not receive HET0016 or vehicle treatment. It is unknown if HET0016 in the absence of injury, such as the control groups, could produce effects on microglial activity. Furthermore, it would be important to evaluate any potential detrimental effects of vehicle treatment in the developing brain. However, this seems unlikely, as the literature does not support neurotoxicity of (2-hydroxypropyl)- $\beta$-cyclodextrin in vitro or in vivo [46, 47], including neonatal piglets [22]. It should also be appreciated that the novel object recognition cognitive results, which can partially depend on hippocampal function, may not necessarily translate to human brain wherein the hippocampus is more ventrally located. Finally, this study was conducted in male rats only. Given the emerging role of sex as a biologic variable, future studies will directly compare both the post-injury response and the response to HET0016 in male versus female rat pups.

\section{Conclusions}

Our data show that administration of a 20-HETE synthesis inhibitor soon after TBI ameliorates tissue loss in immature brain in a sustained manner and results in im- 
proved sensorimotor and cognitive function later in development. The protective mechanism may involve a more rapid progression of microglia to a reparative phenotype. Our results suggest that inhibiting the early generation and action of 20-HETE can significantly ameliorate acute and chronic neuroinflammation and may provide a mechanistic connection between early and chronic neurodegeneration.

\section{Acknowledgments}

The authors express their appreciation to Claire Levine for her expert editorial assistance.

\section{Statement of Ethics}

All animal experiments conform to internationally accepted standards and were approved by the Animal Care and Use Committee of the Johns Hopkins University. Experiments were performed in accordance with National Institutes of Health guidelines.

\section{Disclosure Statement}

The authors have no disclosures.

\section{Funding Sources}

This work was funded by Chongqing Science and Technology Commission grants of China (no. cstc2015jcyjA10095); National Institutes of Health grants NS092747 (C.L.R.); and by the Johns Hopkins Institute for Clinical and Translational Research (ICTR) ATIP Grant (C.L.R.).

\section{Author Contributions}

S.S.: conducted all CCI surgeries, processed and analyzed tissue for histologic analyses, and analyzed and interpreted all data. She also prepared the original manuscript draft and participated in revisions. Z.Z. and S.K.: participated in aspects of the study related to neuroinflammation, including immunohistochemical analysis, cytokine PCR, and microglial cell culture work. Drs. Zhi Zhang and Sujatha Kannan: also participated in manuscript revisions. D.S. and E.K.: performed and analyzed results from the neurologic outcomes tests. K.H. and S.B.-F.: assisted with the microglial cell culture and TNF- $\alpha$ measurement. R.C.K.: supervised and instructed Dr. Shiyu Shu on all aspects of experimental studies. Dr. Raymond C. Koehler: participated in all revisions of manuscript drafts and assisted with data analysis. C.L.R.: instructed Dr. Shiyu Shu on all aspects of CCI surgery, outcome testing, and tissue processing for histologic outcomes. Dr. Courtney L. Robertson: also participated in revisions of all manuscript drafts and in data analysis. All authors reviewed and approved the current manuscript version.

\section{References}

1 Keenan HT, Bratton SL. Epidemiology and outcomes of pediatric traumatic brain injury. Dev Neurosci. 2006;28(4-5):256-63.

2 Giza CC, Mink RB, Madikians A. Pediatric traumatic brain injury: not just little adults. Curr Opin Crit Care. 2007 Apr;13(2):143-52.

3 Yeates KO, Swift E, Taylor HG, Wade SL, Drotar D, Stancin T, et al. Short- and longterm social outcomes following pediatric traumatic brain injury. J Int Neuropsychol Soc. 2004 May;10(3):412-26.

4 Rowe RK, Ziebell JM, Harrison JL, Law LM, Adelson PD, Lifshitz J. Aging with Traumatic Brain Injury: Effects of Age at Injury on Behavioral Outcome following Diffuse Brain Injury in Rats. Dev Neurosci. 2016;38(3):195-205.

5 Loane DJ, Stoica BA, Faden AI. Neuroprotection for traumatic brain injury. Handb Clin Neurol. 2015;127:343-66.

6 Robertson CL, Fidan E, Stanley RM, Noje C, Bayir $\mathrm{H}$. Progesterone for neuroprotection in pediatric traumatic brain injury. Pediatr Crit Care Med. 2015 Mar; 16(3):236-44.

7 Robertson CL, Soane L, Siegel ZT, Fiskum G. The potential role of mitochondria in pediatric traumatic brain injury. Dev Neurosci. 2006;28(4-5):432-46.
8 Li N, Yang Y, Glover D, Zhang J, Saraswati M, Robertson CL, et al. Evidence for impaired plasticity after traumatic brain injury in the developing brain. J Neurotrauma. 2014 Feb; 31(4):395-403.

9 Giza CC, Prins ML. Is being plastic fantastic? Mechanisms of altered plasticity after developmental traumatic brain injury. Dev Neurosci. 2006;28(4-5):364-79.

10 Goodus MT, Guzman AM, Calderon F, Jiang Y, Levison SW. Neural stem cells in the immature, but not the mature, subventricular zone respond robustly to traumatic brain injury. Dev Neurosci. 2015;37(1):29-42.

11 Bell MJ, Kochanek PM. Pediatric traumatic brain injury in 2012: the year with new guidelines and common data elements. Crit Care Clin. 2013 Apr;29(2):223-38.

12 Renic M, Klaus JA, Omura T, Kawashima N, Onishi M, Miyata N, et al. Effect of 20-HETE inhibition on infarct volume and cerebral blood flow after transient middle cerebral artery occlusion. J Cereb Blood Flow Metab. 2009 Mar;29(3):629-39.

13 Jenkins CM, Cedars A, Gross RW. Eicosanoid signalling pathways in the heart. Cardiovasc Res. 2009 May;82(2):240-9.
14 Hoff U, Lukitsch I, Chaykovska L, Ladwig M, Arnold C, Manthati VL, et al. Inhibition of 20-HETE synthesis and action protects the kidney from ischemia/reperfusion injury. Kidney Int. 2011 Jan;79(1):57-65.

15 Roman RJ. P-450 metabolites of arachidonic acid in the control of cardiovascular function. Physiol Rev. 2002 Jan;82(1):131-85.

16 Gebremedhin D, Lange AR, Lowry TF, Taheri MR, Birks EK, Hudetz AG, et al. Production of 20-HETE and its role in autoregulation of cerebral blood flow. Circ Res. 2000 Jul;87(1): 60-5.

17 Dunn KM, Renic M, Flasch AK, Harder DR, Falck J, Roman RJ. Elevated production of 20 HETE in the cerebral vasculature contributes to severity of ischemic stroke and oxidative stress in spontaneously hypertensive rats. Am J Physiol Heart Circ Physiol. 2008 Dec; 295(6):H2455-65.

18 Renic M, Kumar SN, Gebremedhin D, Florence MA, Gerges NZ, Falck JR, et al. Protective effect of 20-HETE inhibition in a model of oxygen-glucose deprivation in hippocampal slice cultures. Am J Physiol Heart Circ Physiol. 2012 Mar;302(6):H1285-93. 
19 Zhu J, Wang B, Lee JH, Armstrong JS, Kulikowicz E, Bhalala US, et al. Additive Neuroprotection of a 20-HETE Inhibitor with Delayed Therapeutic Hypothermia after HypoxiaIschemia in Neonatal Piglets. Dev Neurosci. 2015;37(4-5):376-89.

20 Gebremedhin D, Zhang DX, Carver KA, Rau N, Rarick KR, Roman RJ, et al. Expression of CYP 4A $\omega$-hydroxylase and formation of 20-hydroxyeicosatetreanoic acid (20-HETE) in cultured rat brain astrocytes. Prostaglandins Other Lipid Mediat. 2016 Jul;124:16-26.

21 Kawasaki T, Marumo T, Shirakami K, Mori T, Doi H, Suzuki M, et al. Increase of 20-HETE synthase after brain ischemia in rats revealed by PET study with 11C-labeled 20-HETE synthase-specific inhibitor. J Cereb Blood Flow Metab. 2012 Sep;32(9):1737-46.

22 Yang ZJ, Carter EL, Kibler KK, Kwansa H, Crafa DA, Martin LJ, et al. Attenuation of neonatal ischemic brain damage using a 20 HETE synthesis inhibitor. J Neurochem. 2012 Apr;121(1):168-79.

23 Zhang H, Falck JR, Roman RJ, Harder DR, Koehler RC, Yang ZJ. Upregulation of 20HETE Synthetic Cytochrome P450 Isoforms by Oxygen-Glucose Deprivation in Cortical Neurons. Cell Mol Neurobiol. 2017 Oct; 37(7):1279-86.

24 Miyata N, Seki T, Tanaka Y, Omura T, Taniguchi K, Doi M, et al. Beneficial effects of a new 20-hydroxyeicosatetraenoic acid synthesis inhibitor, TS-011 [N-(3-chloro-4-morpholin-4-yl) phenyl-N'-hydroxyimido formamide], on hemorrhagic and ischemic stroke. J Pharmacol Exp Ther. 2005 Jul; 314(1):77-85.

25 Omura T, Tanaka Y, Miyata N, Koizumi C, Sakurai T, Fukasawa M, et al. Effect of a new inhibitor of the synthesis of 20-HETE on cerebral ischemia reperfusion injury. Stroke. 2006 May;37(5):1307-13.

26 Takeuchi K, Renic M, Bohman QC, Harder DR, Miyata N, Roman RJ. Reversal of delayed vasospasm by an inhibitor of the synthesis of 20-HETE. Am J Physiol Heart Circ Physiol. 2005 Nov;289(5):H2203-11.

27 Roman RJ, Renic M, Dunn KM, Takeuchi K, Hacein-Bey L. Evidence that 20-HETE contributes to the development of acute and delayed cerebral vasospasm. Neurol Res. 2006 Oct;28(7):738-49.
28 Homayoun P, Rodriguez de Turco EB, Parkins NE, Lane DC, Soblosky J, Carey ME, et al. Delayed phospholipid degradation in rat brain after traumatic brain injury. J Neurochem. 1997 Jul;69(1):199-205.

29 Pilitsis JG, Coplin WM, O’Regan MH, Wellwood JM, Diaz FG, Fairfax MR, et al. Free fatty acids in cerebrospinal fluids from patients with traumatic brain injury. Neurosci Lett. 2003 Oct;349(2):136-8.

30 Birnie M, Morrison R, Camara R, Strauss KI. Temporal changes of cytochrome P450 (Cyp) and eicosanoid-related gene expression in the rat brain after traumatic brain injury. BMC Genomics. 2013 May; 14:303.

31 Lu L, Wang M, Yuan F, Wei X, Li W. Roles of elevated 20-HETE in the breakdown of blood brain barrier and the severity of brain edema in experimental traumatic brain injury. $\mathrm{Mol}$ Med Rep. 2018 May;17(5):7339-45.

32 Lu L, Wang M, Wei X, Li W. 20-HETE Inhibition by HET0016 Decreases the Blood-Brain Barrier Permeability and Brain Edema After Traumatic Brain Injury. Front Aging Neurosci. 2018 Jul;10:207.

33 Miyata N, Taniguchi K, Seki T, Ishimoto T, Sato-Watanabe M, Yasuda Y, et al. HET0016, a potent and selective inhibitor of 20-HETE synthesizing enzyme. Br J Pharmacol. 2001 Jun;133(3):325-9.

34 Kilbaugh TJ, Bhandare S, Lorom DH, Saraswati M, Robertson CL, Margulies SS. Cyclosporin A preserves mitochondrial function after traumatic brain injury in the immature rat and piglet. J Neurotrauma. 2011 May; 28(5):763-74.

35 Robertson CL, Saraswati M, Fiskum G. Mitochondrial dysfunction early after traumatic brain injury in immature rats. J Neurochem. 2007 Jun;101(5):1248-57.

36 Liu X, Li C, Falck JR, Roman RJ, Harder DR, Koehler RC. Interaction of nitric oxide, 20 HETE, and EETs during functional hyperemia in whisker barrel cortex. Am J Physiol Heart Circ Physiol. 2008 Aug;295(2):H61931.

37 Shaik JS, Poloyac SM, Kochanek PM, Alexander H, Tudorascu DL, Clark RS, et al. 20-Hydroxyeicosatetraenoic Acid Inhibition by HET0016 Offers Neuroprotection, Decreases Edema, and Increases Cortical Cerebral Blood Flow in a Pediatric Asphyxial Cardiac Arrest Model in Rats. J Cereb Blood Flow Metab. 2015 Nov;35(11):1757-63.
38 Liu Y, Wang D, Wang H, Qu Y, Xiao X, Zhu Y. The protective effect of HET0016 on brain edema and blood-brain barrier dysfunction after cerebral ischemia/reperfusion. Brain Res. 2014 Jan;1544:45-53.

39 Poloyac SM, Zhang Y, Bies RR, Kochanek PM, Graham SH. Protective effect of the 20 HETE inhibitor HET0016 on brain damage after temporary focal ischemia. J Cereb Blood Flow Metab. 2006 Dec;26(12):1551-61.

40 Kehl F, Cambj-Sapunar L, Maier KG, Miyata N, Kametani S, Okamoto H, et al. 20-HETE contributes to the acute fall in cerebral blood flow after subarachnoid hemorrhage in the rat. Am J Physiol Heart Circ Physiol. 2002 Apr;282(4):H1556-65.

41 Pullela R, Raber J, Pfankuch T, Ferriero DM, Claus CP, Koh SE, et al. Traumatic injury to the immature brain results in progressive neuronal loss, hyperactivity and delayed cognitive impairments. Dev Neurosci. 2006; 28(4-5):396-409.

42 Loane DJ, Kumar A, Stoica BA, Cabatbat R Faden AI. Progressive neurodegeneration after experimental brain trauma: association with chronic microglial activation. J Neuropathol Exp Neurol. 2014 Jan;73(1):14-29.

43 Smith C, Gentleman SM, Leclercq PD, Murray LS, Griffin WS, Graham DI, et al. The neuroinflammatory response in humans after traumatic brain injury. Neuropathol Appl Neurobiol. 2013 Oct;39(6):654-66.

44 Byrnes KR, Loane DJ, Stoica BA, Zhang J, Faden AI. Delayed mGluR5 activation limits neuroinflammation and neurodegeneration after traumatic brain injury. J Neuroinflammation. 2012 Feb;9:43.

45 Ramlackhansingh AF, Brooks DJ, Greenwood RJ, Bose SK, Turkheimer FE, Kinnunen $\mathrm{KM}$, et al. Inflammation after trauma: microglial activation and traumatic brain injury. Ann Neurol. 2011 Sep;70(3):374-83.

46 Yaksh TL, Jang JD, Nishiuchi Y, Braun KP, Ro SG, Goodman M. The utility of 2-hydroxypropyl-beta-cyclodextrin as a vehicle for the intracerebral and intrathecal administration of drugs. Life Sci. 1991;48(7):623-33.

47 Abrams DJ, Zheng L, Choo KS, Yang JJ, Wei W, Anchordoquy TJ, et al. An initial animal proof-of-concept study for central administration of clozapine to schizophrenia patients. Schizophr Res. 2008 Mar;100(1-3):86-96. 